\title{
Quantum Cascade Laser Design for Tunable Output at Characteristic Wavelengths in the Mid-Infrared Spectral Range
}

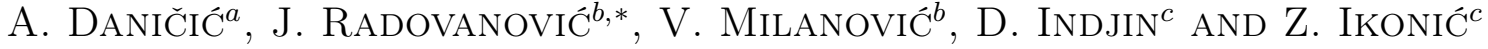 \\ "Vinča" Institute of Nuclear Sciences, PO Box 522, Belgrade, Serbia \\ ${ }^{b}$ Faculty of Electrical Engineering, University of Belgrade, Serbia \\ ${ }^{c}$ Institute of Microwave and Photonics, School of Electronic and Electrical Engineering \\ University of Leeds, UK
}

\begin{abstract}
We present a method for systematic optimization of quantum cascade laser active region, based on the use of the genetic algorithm. The method aims at obtaining a gain-maximized structure, designed to emit radiation at specified wavelengths suitable for direct absorption by pollutant gasses present in the ambient air. After the initial optimization stage, we introduce a strong external magnetic field to tune the laser output properties and to slightly modify the emission wavelength to match the absorption lines of additional compounds. The magnetic field is applied perpendicularly to the epitaxial layers, thus causing two-dimensional continuous energy subbands to split into series of discrete Landau levels. This affects all the relevant relaxation processes in the structure and consequently the lifetime of carriers in the upper laser level. Furthermore, strong effects of band nonparabolicity result in subtle changes of the lasing wavelength at magnetic fields which maximize the gain, thus providing a path for fine-tuning of the output radiation properties. Numerical results are presented for $\mathrm{GaAs}_{3} / \mathrm{Al}_{x} \mathrm{Ga}_{1-x} \mathrm{As}_{\mathrm{s}}$ based quantum cascade laser structures designed to emit at particular wavelengths in the mid-infrared part of the spectrum.
\end{abstract}

PACS numbers: 72.10.-d, 73.21.Fg

\section{Introduction}

Quantum Cascade Laser (QCL) is a unipolar multilayered semiconductor emitter [1] which represent current state-of-the-art among devices operating in the midand far-infrared spectral range. Its emission wavelength can be tuned by designing the band structure, and so far, tailoring of the active band profile has allowed to reach laser wavelengths from $3 \mu \mathrm{m}$ up to $250 \mu \mathrm{m}$. The speedy transfer of QCLs out of the research laboratories and into practical fabrication was stimulated by variety of performances that these devices can deliver in fields as diverse as environmental monitoring, health and safety, security, defense and medical diagnostics, electronic counter measures, chemical sensing [2-6]. Within this last area (chemical detection and monitoring) a large improvement is expected since the combination of QCLs and recent gas sensor developments promise to deliver new levels of spectroscopic performance in terms of detection sensitivity and selectivity [6]. This is due to the fact that many chemical species have very strong characteristic absorption lines in the infrared part of the spectrum.

\footnotetext{
* corresponding author; e-mail: radovanovic@etf.bg.ac.rs
}

In the mid-infrared (MIR) range, the best performance is achieved with GaInAs/AlInAs quantum cascade lasers $[7,8]$, including room temperature continuous wave operation. The performance of GaAs/AlGaAs MIR QCLs is indisputably behind that of $\mathrm{InP}$ based devices (e.g. GaAs has an intrinsic lower gain than GaInAs due to larger effective mass), but they offer other advantages which are attainable with a GaAs-based technology. A very important among these is the use of mature processing techniques developed for GaAs, and less so for InP-based devices $[9,10]$. In this paper, we consider the optimization of the active region of $\mathrm{GaAs} / \mathrm{Al}_{x} \mathrm{Ga}_{1-x}$ As based midinfrared QCL, with the goal of maximizing the output properties at characteristic wavelengths, suitable for absorption by pollutant gasses, such as $\mathrm{SO}_{2}, \mathrm{HNO}_{3}, \mathrm{CH}_{4}$, $\mathrm{NH}_{3}$, etc. The GaAs/AlGaAs system was chosen because it is well understood from the material-growth point of view, and is virtually strain-free. In addition, the material parameters relevant for the effects considered in this work (especially the band nonparabolicity coefficients) are known more accurately, however, the presented numerical procedure is practically independent of the material system.

Among the goals of calculations presented in this work is to demonstrate the possibility of achieving several pre- 
defined wavelengths with a single structure by variation of the magnetic field. The parameters of interest in the calculation of the optical gain, e.g. the population inversion and the transition matrix element, depend via the wave functions on the potential profile which may be varied to optimize the performance of the structure. However, the relationships between these parameters are very complex, making the optimization process difficult and demanding so it becomes apparent that some carefully selected optimization technique for tailoring of structural profile should be employed [11]. The procedure adopted here relies on applying an elaborate tool for global optimization, namely the Genetic Algorithm (GA), whose inherent parallelism in generating and processing the trial solutions allows us to deal with complex optimization problems [12].

Magnetic field is an efficient spectroscopic tool for studying the scattering mechanisms in the QCL active region [13]. Since the energy and length scales of the spatial and magnetic confinement are similar, application of a strong magnetic field has proven as a sensitive instrument to study and control fundamental processes in the physics of intersubband transitions - quantum confinement and intersubband relaxation. An intense magnetic field applied perpendicular to the 2DEG planes of a QCL causes two-dimensional continuous energy subbands to split into series of discrete Landau levels (LLs). Since the arrangement of Landau levels depends strongly on the magnitude of the magnetic field, this enables one to control the population inversion in the active region, and hence the optical gain [14-17]. Furthermore, strong effects of band nonparabolicity result in subtle changes of the lasing wavelength at magnetic fields which maximize the gain, thus providing a path for fine-tuning of the output radiation. The complete procedure is illustrated by performing two sets of numerical calculations for quantum cascade lasers designed to emit at specified wavelengths in the mid-infrared spectral range. The magnetic fields required to achieve sufficient wavelength tunability of the optimized QCL structures obtained in this work are (regrettably) found to be extremely high (20-60 T). Such values are indeed unacceptable within the present-day technology and restrict practical application of the particular structures. However, the results point to the possibility of magnetic field assisted realization of multiple emission wavelengths, and the future work will focus on implementing the theory and optimization procedure presented in this paper, to the design of QCLs in which the tunability may be achieved with technologically feasible and realistic magnetic fields.

\section{Theoretical consideration}

The active region of the QCL structure under consideration comprises three coupled quantum wells (QWs) biased by an external electric field $K$, as displayed in Fig. 1. This system has three energy states, i.e., subbands $(n=1,2,3)$, and the laser transition occurs between subbands $n=3$ and $n=2$. The energy difference between the two lower subbands should match the LOphonon energy, in order to ensure fast depopulation of the lower state of the laser transition via resonant optical-phonon emission. This active region is surrounded by suitable emitter/collector regions in form of superlattices, designed as Bragg reflectors, which inject electrons into state $n=3$ on one side, and allow for rapid extraction of carriers from the lowest subband on the other side. These two mechanisms are responsible for achieving population inversion in the structure.

The electronic subbands from Fig. 1 have free particle-like energy dispersion in the direction parallel to the QW planes: $E_{\|}=\hbar^{2} k_{\|}^{2} / 2 m^{*}$, where $m^{*}$ is the effective mass, and $k_{\|}$is the in-plane wave vector. The nonradiative lifetime for the state $\left|3, k_{\|}\right\rangle$is limited by electron-LO-phonon scattering into the two lower subbands of the active region, and the optical gain reads

$$
\begin{aligned}
g_{3,2} & =\frac{e^{2}}{2 n \varepsilon_{0} \omega m_{0}^{2} c} \\
& \times \int_{0}^{+\infty} F_{3,2} P_{3,2}^{2} \delta\left(E_{3}-E_{2}-\hbar \omega\right) \mathrm{d}\left(k_{\|}^{2}\right)
\end{aligned}
$$

where $n$ is the refractive index, $\varepsilon_{0}$ is the vacuum dielectric permittivity, $c$ is the speed of light in vacuum, $F_{3,2}$ stands for the difference of Fermi-Dirac functions for the initial and the final state, while $P_{3,2}$ is the momentum matrix element.

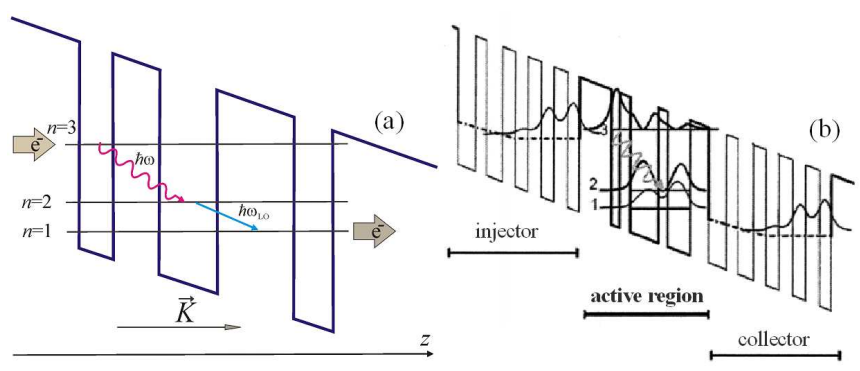

Fig. 1. A schematic diagram of (a) the QCL active region described in the main text and (b) one and a half period of QCL structure, comprising the active region and suitable injector/collector layers.

When the structure from Fig. 1a is subjected to a strong magnetic field $B$ in the $z$-direction, continuous subbands $E_{n}\left(k_{\|}\right)$transform into series of individual (strictly discrete) states at energies $E_{n, l} \approx E_{n}+$ $(l+1 / 2) \hbar \omega_{c}$, where $l=0,1,2, \ldots$ is the Landau index and $\omega_{c}=e B / m^{*}$ is the cyclotron frequency. Variation of the magnetic field $B$ influences the configuration (energy spacings) of the discrete states, and hence the probabilities for emission of LO phonons and the optical gain. The values of $B$ which give rise to resonant LO phonon emission are found by solving the equation: $E_{3,0}-E_{n, l}=\hbar \omega_{\mathrm{LO}}($ with $n=1,2)$, where $\hbar \omega_{\mathrm{LO}}$ is the LO phonon energy.

Optical transitions in this system are allowed only between states with the same value of the Landau index, i.e. 
$(i, l) \rightarrow(f, l)$. The fractional absorption (or, if it comes out to be negative, the gain) on transitions corresponding to the lasing energy, i.e. $(2, l) \rightarrow(3, l)$, reads $[15,16]$ :

$$
A_{2, l \rightarrow 3, l}=\frac{2 e^{3} \pi B}{\hbar n \varepsilon_{0}} \frac{d_{2,3}^{2}}{\lambda} \delta\left(E_{3, l}-E_{2, l}-\hbar \omega\right) F_{2, l ; 3, l} .
$$

Here, $d_{i f}=\left\langle\eta_{i}|z| \eta_{f}\right\rangle$ is the transition matrix element, and $\eta_{i}$ and $\eta_{f}$ denote the $z$-dependent parts of the wavefunctions. Using the expression for the electron areal density in the state $(n, l)$, i.e. $N_{n, l}=e B /(\pi \hbar) F_{\mathrm{FD}}\left(E_{n, l}\right)$, and summing over all LLs, we get the total gain on all transitions between LLs belonging to subbands $n=3$ and $n=2$ of the QCL active region $[15,16]$ :

$$
g_{3,2}=\frac{2 e^{2} \pi^{2}}{n \varepsilon_{0}} \frac{d_{2,3}^{2}}{\lambda} \delta\left(E_{3, l}-E_{2, l}-\hbar \omega\right)\left(N_{\mathrm{S} 3}-N_{\mathrm{S} 2}\right),
$$

where $N_{\mathrm{Sn}}=\sum_{l} N_{n, l}$. To determine the population inversion $N_{\mathrm{S} 3}-N_{\mathrm{S} 2}$, one has to find the electron distribution over all the states in the active region. This is obtained by solving the system of rate equations, which describe the change in level population as the difference between the rate at which the carriers arrive and the rate at which they leave. The exact form of the rate equations used in this work is presented in detail in references $[15,16]$. We should note, however, that these rate equations assume perfect injection from the injector to the upper laser level, but there have been some other works on the transport in quantum cascade lasers in a magnetic field which avoid such an assumption, either within the rate equation formalism or within the density matrix formalism [18-21].

\section{Numerical results}

In order to optimize the laser performance at selected wavelength, one must consider the entire free-parameters space and this type of search is best completed by some established method for global optimization, such as the genetic algorithm employed in this work [12]. This algorithm is inspired by the theory of evolution, where problems are solved by selecting the best fit solution which is then allowed to survive. GA begins with a set of solutions called population, which is used as a basis for generating another set of offsprings with best possible characteristics (defined by the value of the objective, i.e. the fitness function). This process is repeated until some predefined criterion is met (this could be, e.g. the total number of populations generated or the improvement in the fittest solution). One of the most important phases in the implementation of GA algorithm is the selection of a formal fitness function, which should be defined so to encompass all the goals of optimization. Here, the objective is to optimize the optical gain at selected wavelength, hence the fitness function is taken as:

$$
\begin{aligned}
F & =g(B=0) /\left\{\left[\left(E_{3}-E_{2}-\hbar \omega\right)^{2}+\Theta^{2}\right]\right. \\
& \left.\times\left[\left(E_{2}-E_{1}-\hbar \omega_{\mathrm{LO}}\right)^{2}+\Theta^{2}\right]\right\},
\end{aligned}
$$

where the term in the denominator favours achieving specified emission energy $\hbar \omega$, as well as the LO-phonon resonance. In addition, $\Theta$ is a non-zero constant, which ensures that $F$ is strongly driven towards resonance in the course of optimization, while remaining finite at the exact resonance, and $g(B=0)$ is the optical gain in the absence of the magnetic field, given by Eq. (1). In numerical calculation, the optical gain can be expressed via the gain coefficient $g^{*}=\left(1-\tau_{21} / \tau_{32}\right) \tau_{3} d_{32}^{2}$, where $\tau_{21}$ and $\tau_{32}$ are the scattering times and $\tau_{3}$ is the upper laser level lifetime [22].

We have optimized the QCL active region at two specific wavelengths: one is at $\lambda \approx 10.3 \mu \mathrm{m}$ and corresponds to a characteristic line in the spectrum of ammonia, and another at $\lambda \approx 7.3 \mu \mathrm{m}$, suitable for detection of sulphur-dioxide presence [23]. The structural parameters obtained by GA in the first case read: $19 \AA$, $37 \AA, 40 \AA, 23 \AA, 39 \AA$ (for the well and the barrier widths, respectively, going from left to right) and $U_{b}=0.3175 \mathrm{eV}$ (the barrier height), which corresponds to aluminum mole fraction of $38 \%$, so the structure may be realized by $\mathrm{GaAs} / \mathrm{Al}_{0.38} \mathrm{Ga}_{0.62} \mathrm{As}$. The material parameters used in calculations are: $m^{*}=0.0665 m_{0}$ ( $m_{0}$ is the free electron mass), $n=3.3$, and the conduction band discontinuity between GaAs and AlAs is $\Delta E_{c}=0.8355 \mathrm{eV}$. The applied electric field in the $\mathrm{z}$ direction is $K=48 \mathrm{kV} / \mathrm{cm}$, and the minima of energy subbands are at $E_{1}\left(k_{\|}=0\right)=0.0767 \mathrm{eV}, E_{2}\left(k_{\|}=\right.$ $0)=0.1125 \mathrm{eV}$ and $E_{3}\left(k_{\|}=0\right)=0.2325 \mathrm{eV}$, as presented in Fig. 2 (a), together with the corresponding wavefunctions. The calculated optical gain per unit of injection current $\left(g=g_{32} / J\right)$ is $g=4.32 \times 10^{-3} \mathrm{~cm}^{2} / k A$. Another set of calculations was performed by setting $E_{32}=170 \mathrm{meV}$ in the fitness function (4) for the genetic algorithm, and the optimization procedure has delivered a structure displayed in Fig. 2 (b), with the following layer widths: $19 \AA, 11 \AA, 32 \AA, 39 \AA, 23 \AA$, $38 \AA$ (starting from the left well) and the barrier heights $U_{b}=0.3175 \mathrm{eV}$, leading to the same material combination $\left(\mathrm{GaAs} / \mathrm{Al}_{0.38} \mathrm{Ga}_{0.62} \mathrm{As}\right)$ as in the previous numerical example The optical gain per unit of injection current now amounts to $g=1.27 \times 10^{-3} \mathrm{~cm}^{2} / k A$.
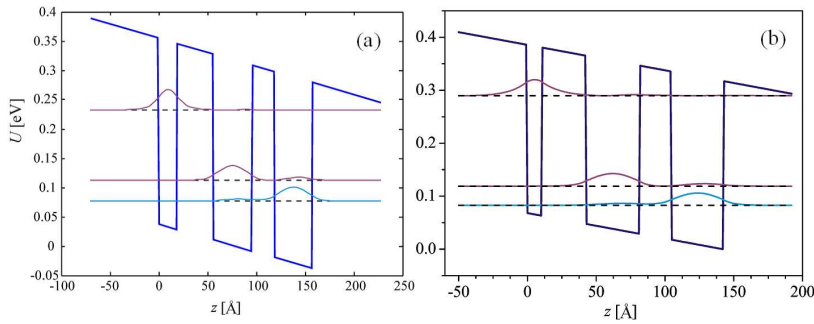

Fig. 2. (a) The active region of QCL, optimized for emission at $\lambda \approx 10.3 \mu \mathrm{m}$ which corresponds to a specific line in the spectrum of $\mathrm{NH}_{3}$. (b) The active region of QCL, optimized for emission at $\lambda \approx 7.3 \mu \mathrm{m}$ which corresponds to a characteristic line in the spectrum of $\mathrm{SO}_{2}$.

As already pointed out, when this structure is placed in a strong external magnetic field in the $z$-direction, 
the $2 \mathrm{D}$ subbands $E_{n 0}+\hbar^{2} k_{\|}^{2} / 2 m_{\| n}\left(E_{n 0}\right)$ split into series of discrete LLs, the energies of which (with band nonparabolicity included) are given by [24]: $E_{n, l}=$ $E_{n 0}+(l+1 / 2) \hbar e B / m_{\| n}\left(E_{n 0}\right)-1 / 8\left[\left(8 l^{2}+8 l+5\right) \alpha_{1}^{\prime}+\left(l^{2}+\right.\right.$ $\left.l+1) \beta_{1}^{\prime}\right]\left(\hbar e B / m^{*}\right)^{2}$. The in-plane electron effective mass is here calculated as $m_{\|}\left(E_{n 0}\right)=m^{*}\left[1+\left(2 \alpha_{1}^{\prime}+\beta_{1}^{\prime}\right) E_{n 0}\right]$, $[15,16$,$] , where the nonparabolicity parameters \alpha_{1}^{\prime}$ and $\beta_{1}^{\prime}$ are evaluated according to Ref. [24]. It is evident that the realistic effects of band nonparabolicity influence the energy separation between the levels relevant for the radiative transition, and this allows for the shift of the emission wavelength by variations of the magnetic field. The effects of magnetic field (in the range 20-60 T) on the optical gain in structures from Fig. 2 are presented in Figs. 3a and b, respectively. Such high values of the magnetic field are necessary to provide sufficient changes in the lasing energy. Oscillations of the gain with $B$ are very pronounced, and prominent peaks are found at values of the magnetic field for which the resonant emission of LO phonons is inhibited so the lifetime of carriers in the upper laser state is increased.

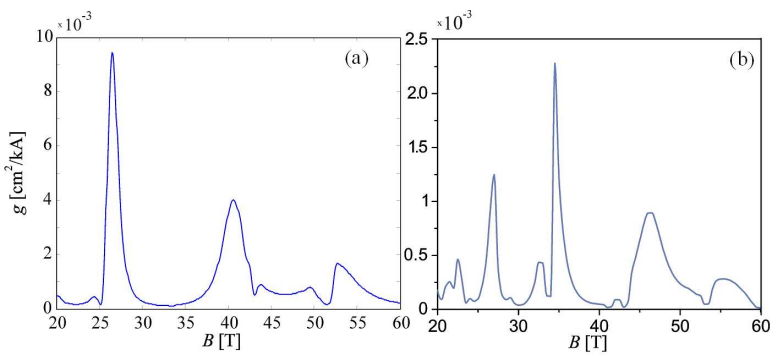

Fig. 3. The optical gain (per unit of injection current) as a function of the applied magnetic field, at $T=300 \mathrm{~K}$, (a) for the structure from Fig. 2a, optimized at $\lambda \approx 10.3 \mu \mathrm{m}$, (b) for the structure from Fig. $2 \mathrm{~b}$, optimized at $\lambda \approx 7.3 \mu \mathrm{m}$.

For the structure from Fig. 2a, the maximum of optical gain per unit of injection current is obtained at $B=26.5 \mathrm{~T}$, where the transition wavelength is shifted to $\lambda=10.6 \mu \mathrm{m}$, matching an absorption line in the spectrum of ethylene $\left(\mathrm{C}_{2} \mathrm{H}_{4}\right)$, while the peak second in magnitude, at $B=40.5 \mathrm{~T}$, may be used to monitor the presence of sulphur-hexafluoride $\left(\mathrm{SF}_{6}\right)$ at $k=930 \mathrm{~cm}^{-1}$ [23]. On the other hand, the active region from Fig. $2 \mathrm{~b}$ exhibits maximal gain at $B=34.5 \mathrm{~T}$, and the lasing energy in that case corresponds to a characteristic absorption line in the spectrum of $\mathrm{HNO}_{3},(\lambda=7.52 \mu \mathrm{m}$, i.e. the wavenumber $k=1330 \mathrm{~cm}^{-1}$ [23]). Another peak of the gain is reached at magnetic field value of $B=46 \mathrm{~T}$ and the energy between the laser levels in this case reads $E_{3,0}-E_{2,0}=163.8 \mathrm{meV}$, which coincides with the line at $k=1320 \mathrm{~cm}^{-1}$ in the spectrum of methane $\left(\mathrm{CH}_{4}\right),[23]$

\section{Conclusion}

In this paper we have focused on the systematic optimization and wavelength tunability of quantum cas- cade laser emitting at wavelengths specified according to absorption fingerprints of particular harmful compounds found in the atmosphere. The presented method relies on application of the genetic algorithm for maximizing the optical gain, and subsequently, it utilizes the additional carrier confinement achieved by introducing strong external magnetic field to the optimized structure. This magnetic field alters the electron energy spectrum and thus provides a mechanism for modulating the laser output properties, including the delicate changes in the lasing wavelength. Numerical results are presented for QCL structures designed to emit at selected wavelengths in the mid-infrared part of the spectrum. However, the procedure can easily be modified for other characteristic wavelengths (pollutant gasses) of interest, simply by changing the objective function for optimization.

\section{Acknowledgments}

This work was supported by the Ministry of Science (Republic of Serbia), ev. no. 141006. and by NATO Collaborative Linkage Grant (reference CBP. EAP.CLG 983316).

\section{References}

[1] J. Faist, F. Cappaso, D.L. Sivco, A.L. Hutchinson, A.Y. Cho, Science 264, 553 (1994).

[2] Y. Bai, S. Slivken, S.R. Darvish, M. Razeghi, Appl. Phys. Lett. 93, 021103 (2008).

[3] A. Kosterev, F. Tittel, IEEE J. Quantum Electron. 38, 582 (2002).

[4] G. Wysocki, R. Lewicki, R.F. Curl, F.K. Tittel, L. Diehl, F. Capasso, M. Troccoli, G. Hofler, D. Bour, S. Corzine, R. Maulini, M. Giovannini, J. Faist, Appl. Phys. B 92, 305 (2008).

[5] J.B. McManus, J.H. Shorter, D.D. Nelson, M.S. Zahniser, D.E. Glenn, R.M. McGovern, Appl. Phys. B 92, 387 (2008).

[6] E. Normand, I. Howieson, M. McCulloch, P. Black, Proc. SPIE 6402, 2006 p. 64020G.

[7] A. Vasanelli, A. Leuliet, C. Sirtori, A. Wade, G. Fedorov, D. Smirnov, G. Bastard, B. Vinter, M. Giovannini, J. Faist, Appl. Phys. Lett. 89, 172120 (2006).

[8] L. Diehl, D. Bour, S. Corzine, J. Zhu, G. Höfler, M. Lončar, M. Troccoli, F. Capasso, Appl. Phys. Lett. 88, 201115 (2006).

[9] C. Sirtori, H. Page, C. Becker, Phil. Trans. R. Soc. Lond. A 359, 505 (2001).

[10] M. Garcia, E. Normand, C.R. Stanley, C.N. Ironside, C.D. Farmer, G. Duxbury, N. Langford, Opt. Commun. 226, 39 (2003).

[11] S. Tomić, M. Tadić, V. Milanović, Z. Ikonić, J. Appl. Phys. 87, 7965 (2000).

[12] J. Radovanović, V. Milanović, Z. Ikonić, D. Indjin, J. Phys. D, Appl. Phys. 40, 5066 (2007).

[13] A. Leuliet, A. Vasanelli, A. Wade, G. Fedorov, D. Smirnov, G. Bastard, C. Sirtori, Phys. Rev. B 73, 085311 (2006). 
[14] C. Becker, C. Sirtori, O. Drachenko, V. Rylkov, J. Leotin, Appl. Phys. Lett. 81, 2941 (2002).

[15] J. Radovanović, A. Mirčetić, V. Milanović, Z. Ikonić, D. Indjin, P. Harrison, R.W. Kelsall, Semicon. Sci. Technol. 21, 215 (2006).

[16] J. Radovanović, V. Milanović, Z. Ikonić, D. Indjin, P. Harrison, J. Appl. Phys. 97, 103109 (2005).

[17] A. Wade, G. Fedorov, D. Smirnov, S. Kumar, B.S. Williams, Q. Hu, J.L. Reno, Nature Photon. 3 41 (2009).

[18] I. Savić, N. Vukmirović, Z. Ikonić, D. Indjin, R.W. Kelsall, P. Harrison, V. Milanović, Phys. Rev. B 76, 165310 (2007).

[19] I. Savić, Z. Ikonić, V. Milanović, N. Vukmirović, V.D. Jovanović, D. Indjin, P. Harrison, Phys. Rev. B 73, 075321 (2006).
[20] I. Savić, Z. Ikonić, N. Vukmirović, D. Indjin, P. Harrison, V. Milanović, Appl. Phys. Lett. 89, 011109 (2006).

[21] D. Indjin, Z. Ikonić, V.D. Jovanović, N. Vukmirović, P. Harrison, R.W. Kelsall, Semicond. Sci. Technol. 20, S237 (2005).

[22] A. Mirčetić, D. Indjin, Z. Ikonić, P. Harrison, V. Milanović, R.W. Kelsall, J. Appl. Phys. 97, 084506 (2005).

[23] www.hitran.com .

[24] U. Ekenberg, Phys. Rev. B 40, 7714 (1989). 